\title{
Leakage of Hepaticojejunal Anastomosis: Reoperation
}

\author{
Ulrich F. Wellner Tobias Keck \\ Clinic for Surgery, University Clinic Schleswig-Holstein Campus Lübeck, Lübeck, Germany
}

\section{Keywords}

Hepaticojejunostomy - Bile leak - Liver resection . Pancreatectomy · Postoperative complications

\section{Summary}

Background: Leakage of a hepaticojejunal anastomosis is a rare event with potential major morbidity. Surgeons must be aware of the technical armamentarium and pitfalls in revisional surgery for hepaticojejunal anastomosis leakage. Methods: Review of the available literature and discussion of technical details based on experience and expert opinion. Results: Early bile leaks as well as failed interventional therapy are indications for reoperation. Almost all reports only mention rates of leakage as a secondary endpoint but do not report details of treatment and outcome. Few authors have compared outcome after reoperation versus interventional approaches in observational studies, with the latter resulting in lower morbidity. The complex and individual situation of the complication makes evidence-based argumentation difficult; this is why personal expert opinions have to be taken into account in this review. The technical aspects and pitfalls of revisional surgery are outlined but represent anecdotal evidence as comparative studies are lacking. Conclusion: Bile leak after bilioenteric anastomosis is a rare condition that requires differentiated workup and therapy. Early leaks usually result from technical problems and are amenable to repair by reoperation. Reoperation technique can only be discussed on a low evidence level.

(c) 2017 S. Karger GmbH, Freiburg

\section{Introduction}

\section{Bilioenteric Anastomoses}

Conditions that require bilioenteric anastomoses include resections of the extrahepatic bile tree for tumors or stenosis, repair of biliary tree injury, and liver transplantation. Hepaticojejunostomy is usually performed in an end-to-side fashion between the common bile duct (CBD) proximal to the level of the cystic duct and a Roux-Y loop of the jejunum. Hepaticoenteric anastomoses to the proximal biliary system are more demanding as several subtle single anastomoses to intrahepatic bile ducts or to the fibrous plate of several ducts have to be performed after extended hepatic resections. While hepaticoduodenostomy is a technical option when the duodenum is not removed, it has been abandoned due to increased cholangitis by reflux of food from the duodenum [1] and potential tension of the anastomosis. When the anastomosis is located proximal to the CBD bifurcation, latero-lateral connection of multiple small bile ducts in a common anastomosis is the preferred technique. Some authors refer to this as hepatoenterostomy or portoenterostomy [2]. Further details are discussed in the following.

\section{Definition and Differential Diagnosis of Bile Leak}

Bile leak is defined by the International Study Group for Liver Surgery as bilious secretion with more than three times the serum concentration of bilirubin on or after day 3 , or necessity of intervention due to collections of biliary fluid [3]. It is important to bear in mind that leakage of biliary secretions may occur from other sites than the bilioenteric anastomosis. In case of liver resection, bile leak typically originates from the parenchymal resection margin [4]. The closed end of the jejunal loop is another rare site of bile leak, as are injuries to the biliary tree and jejunal loop outside of the anastomosis region. Rather rare are combined leaks of bile and pancreatic juice after procedural lacerations of the small bowel after surgery. In the latter cases, amylase or lipase are usually elevated in the drain fluid, too, which makes a separation of these results easy. After pancreatoduodenectomy, biliary leakage may also be derived from an insufficient pancreatojejunostomy, especially

\section{KARGER}

(c) 2017 S. Karger GmbH, Freiburg

Fax +497614520714 
when a single jejunal loop is used for bilioenteric and pancreatoenteric anastomosis [5] and the distance between the pancreatic and the biliary anastomosis is short. Diagnostic efforts for exact localization of the bile leak are therefore mandatory before considering reoperation.

\section{Workup and Indication}

\section{Localization of Bile Leak}

Conventional options include radiographic fistulography by injection of contrast agent via T-drains, intraoperatively placed internal-external biliary drains (Neuhaus drain), or drains remaining after surgery or intervention. A preoperatively or postoperatively inserted external percutaneous transhepatic drain (PTHD) may also serve this diagnostic as well as a therapeutic purpose [6]. Endoscopic retrograde cholangiography can be possible despite Roux-Y reconstruction when push-and-pull endoscopy is performed. Even cholangioscopy is possible via PTHD [7]. Apart from radiographic methods, gadoxetate disodium-enhanced magnetic resonance cholangiography enables non-invasive visualization of bile leakage in the delayed hepatobiliary phase [8]. The authors prefer atraumatic approaches without endoscopy, such as the above-mentioned methods of fistulography, if revisional surgery as a primary method of identifying the leak is not an option (fig. 1).

\section{Indications for Reoperation}

Modern interventional procedures like PTHD nowadays often obviate the need for reoperation [6]. Indications for reoperation of bile leak are early bile leaks (within the first 3 days after the primary operation), failure of interventional therapy, or arrosion hemorrhage not amenable to interventional therapy. We strongly believe that early bile leaks of high volume $(>400 \mathrm{ml})$ should primarily be approached with reoperation within the first 3 days as technical mistakes or ischemic conditions of the biliary duct are likely reasons of early bile leak.

\section{Operation Technique}

\section{Reoperation for Early Bile Leaks}

As already mentioned, early bile leaks (within the first 3 days after operation) are usually a result of technical failure. Possible mechanisms include primary incomplete closure and early suture dehiscence by excessive traction during or after completion of the anastomosis, longitudinal shears of the biliary duct due to too much tension during completion of the anastomosis, or early ischemic conditions of the biliary duct. Interrupted sutures have been reported to be more prone to leak, while running sutures are more likely to result in stenosis of the bilioenteric anastomosis [1]. Comparisons herein remain difficult as a variety of factors remains to be observed, e.g. the diameter of the bile duct, intrahepatic or proximal anastomosis, and the use of atraumatic and thin suture material (PDS 5-0 and 6-0). Reoperation during the early postop-
Fig. 1. Control of a repaired bilioenteric anastomosis. The inserted internal-external drain (Neuhaus drain) is used to control the patency and impermeability of the anastomosis. There is no extravasation of contrast media.

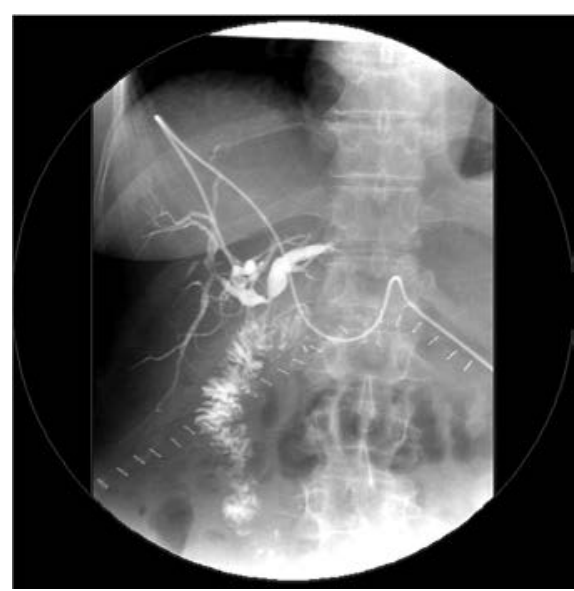

Fig. 2. Gütgemann modification at the common hepatic duct.

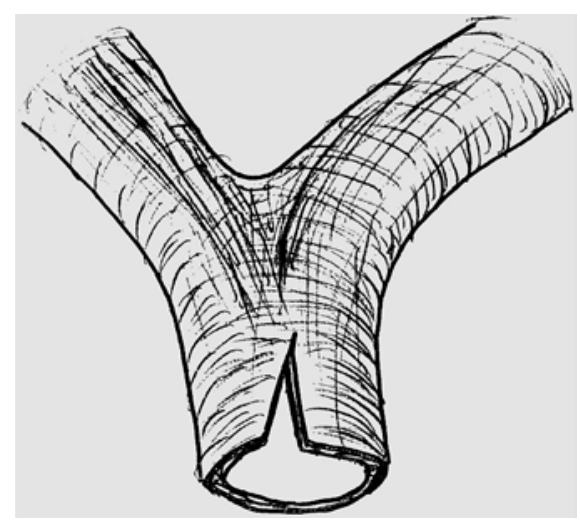

erative period is usually not complicated by postoperative peritoneal adhesions or peritonitis, as biliary peritonitis is a chemical form of peritonitis and requires some time to develop. Associated biliary fluid collections must be evacuated and the biliary anastomosis region must be adequately drained, also in view of possible recurrent leak. In rare instances, direct visualization of the bile leak can be problematic. The so-called White test (injection of propofol into the biliary tree [9]) can help to visualize the leak but requires a placed catheter/drain. Positioning of white cloth to the anastomosis can reveal a minor biliary leak during the time of the operation.

Small leaks resulting from incomplete primary suture can be oversewn. In case of larger defects or ischemia, partial closure with insertion of a T-drain through the anastomotic leak is an option as is the revision of the whole anastomosis and the re-anastomosis in the hilar region for better perfusion. If a T-drain is used, a tight closure of the anastomosis around the T-drain insertion site is necessary to avoid ongoing leakage. For this closure, thin monofilament suture material in a single-stich method is recommended. For semicircumferential or larger defects, resection and re-anastomosis should be chosen in any case.

The anastomosis region critically depends on the proximal blood supply from the right hepatic artery [1]. The biliary tree down to the proximal CBD receives blood from branches of the right hepatic and cystic artery, while the distal CBD is supplied by branches from the gastroduodenal artery and pancreatic head, forming a 'water sheath' 

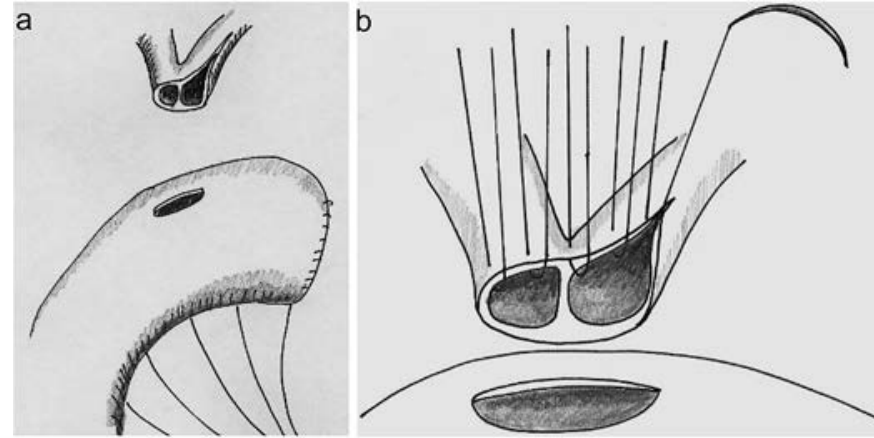

C
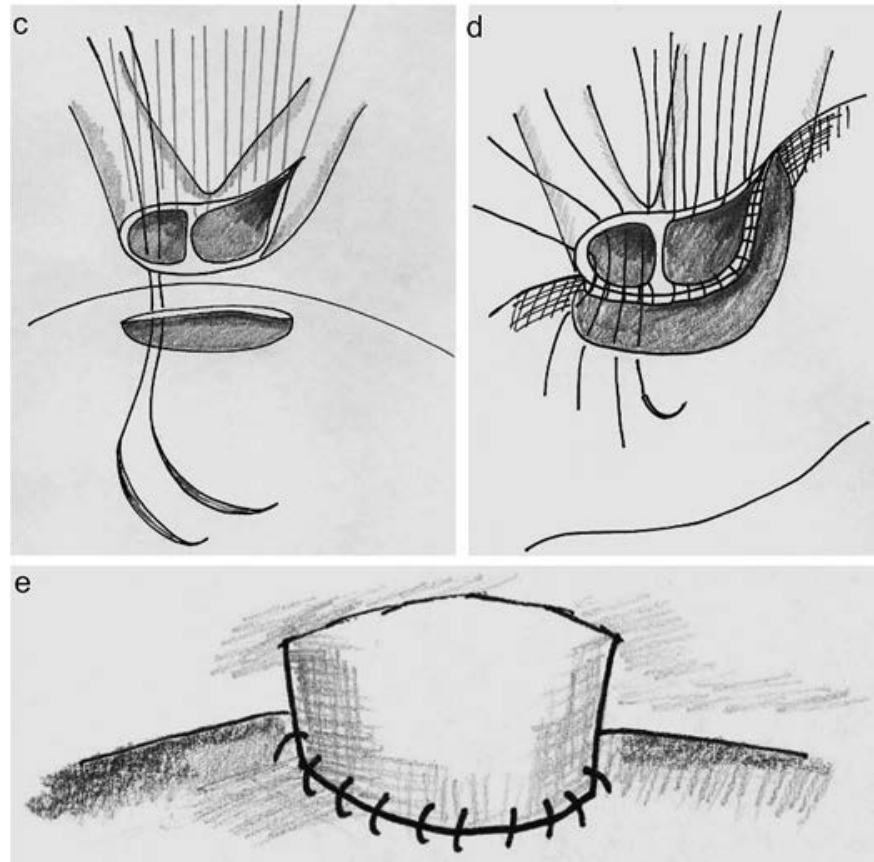

Fig. 3. a-e Steps of the hilar biliary anastomosis by Hepp-Couinaud: Longitudinal extension of the left biliary duct, prepositioning of the sutures of the anterior aspect of the anastomosis with 5-0 or 6-0 double-armed C1 monofilament suture, prepositioning of the posterior aspect of the anastomosis with 5-0 or 6-0 single-armed C1 monofilament sutures, completion of the posterior wall, completion of the anterior wall, completed anastomosis.

at the level of the cystic artery and cystic duct. This anatomical knowledge is necessary in pancreatic surgery and biliary anastomosis where the only blood supply to the biliary duct comes from the (right) hepatic artery after the gastroduodenal artery was divided. To avoid ischemic anastomosis complications, essential principles are to establish the anastomosis proximal to the level of the cystic duct, to avoid excessive denudation of the bile duct proximal to the anastomosis, and to preserve blood flow through the right hepatic artery. This condition has to be evaluated before revisional surgery (e.g. by computed tomography angiography) for bile duct lacerations after cholecystectomy, where accompanying arterial injuries to the right hepatic artery have frequently been described.

In order to repair dehiscent anastomoses or to avoid complications in the first place, a tension-free anastomosis must be established under appropriate vision. Microsurgical technique, interrupted resorbable thin monofilament sutures, and temporary stenting of the anastomosis by internal-external (Neuhaus) drain
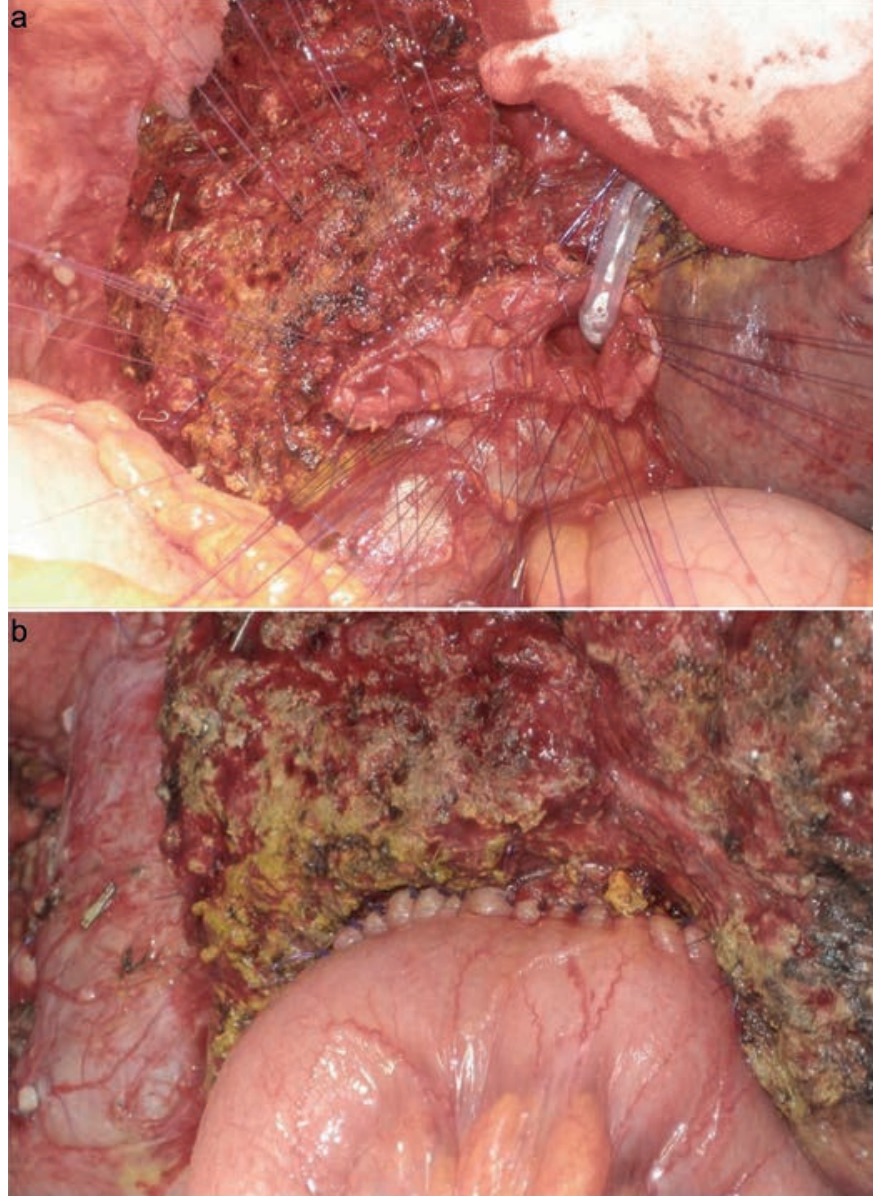

Fig. 4. Hepp-Couinaud technique of hilar hepaticojejunostomy. After resection of the common bile duct up to the level of the bifurcation, hepaticojejunostomy is established by interrupted monofilament (PDS 6-0) sutures joining the left and right hepatic duct in a common anastomosis site. a All sutures are placed before knotting to ensure appropriate vision and an internal-external drain (Neuhaus drain) is placed over the anastomosis to drain the jejunal limb and stent the anastomosis. b Finished anastomosis.

in cases of intrahepatic or problematic anastomoses are recommended, especially for small bile ducts [1].

After resection of the dehiscent anastomosis, re-anastomosis to the proximal $\mathrm{CBD}$ or even segmental bile ducts proximal to the CBD bifurcation might be necessary. In this case, the authors recommend to also opt for an additional reinsertion site at the small bowel and to shorten the small bowel or reinsert the duct in another area and close the primary anastomotic site of the small bowel with a running suture. For a small-caliber CBD, a Gütgemann modification (wedge-shaped excision) (fig. 2) can help to augment the diameter of the anastomosis and provide better visualization. When the resection extends higher up into to the liver hilus, bilioenteric anastomosis has to be performed in a side-toside fashion (Hepp-Couinaud) (fig. 3). Here, the bile duct to be anastomosed is opened in longitudinal direction on its anterior surface to increase the length of the suture line to a technically feasible degree. Usually the left biliary duct has to be opened in this 
Fig. 5. Technical tips for performing a repair after insufficiency of a biliary anastomosis: parachute technique on distance (a), preoperaof vital tissue, resection of non-vital tissue, proximalisation of the anastomosis (a), patic drainage of smaller biliary ducts (intraluminal stent (a)), completion with thin monofilament single sutures in a tension-free method (b). tive focus control, use transenteric or transhe-

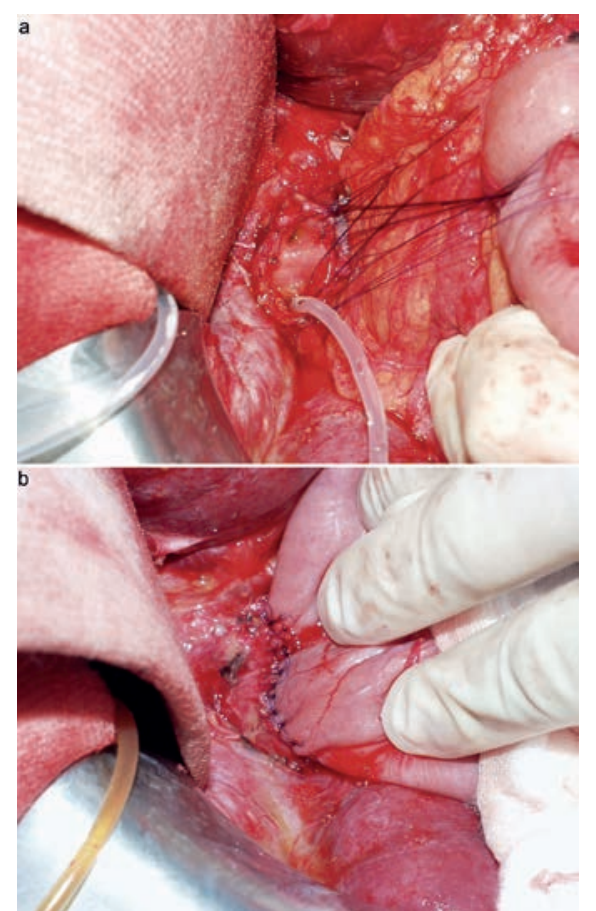

technique as it is smaller in diameter and therefore bears a higher risk of secondary stenosis (www.webop.de/anastomosentechnik-biliodigestiv-nach-hepp-couinaud-60/). Using this technique, not only the CBD bifurcation (fig. 4, 5) but also more proximal segments of the biliary tree can be connected to the jejunum. Incision or resection of liver parenchyma to gain adequate access to the proximal bile ducts might be necessary [10]. In general, proximal anastomosis is technically better feasible to the left hepatic duct due to a longer and more superficial extrahepatic course than the right hepatic duct. Traction must be avoided by adequate mobilization of the jejunal loop in an antecolic or retrocolic fashion.

A rarely employed method to control high-risk anastomoses is the establishment of an inspection jejunostomy in the Roux-Y loop, allowing for easy endoscopic access to the anastomosis [11]. Another possibility of endoscopic anastomosis inspection is percutaneous transhepatic cholangioscopy [7]. Both techniques were described for diagnosis and treatment of ischemic biliary tree strictures, tumor stenosis, or cholelithiasis. Successful reconstruction of the bile duct using an autologous vein graft has been reported anecdotally [12].

\section{Late Bile Leaks}

Bile leaks $\geq 5$ days after the operation can be the result of partial or prolonged ischemia, thermal injury, or septic arrosion of the anastomosis. In this situation, reoperation should remain the treatment of last choice, as peritoneal adhesions and local or diffuse peritonitis complicate the operation and bear significant risk of collateral damage. In these cases, however, adhesions and compartmentation provide protection against diffuse peritonitis when left in place. The treatment of choice in these cases is PTHD or endoscopic retrograde drainage, combined with interventional drainage

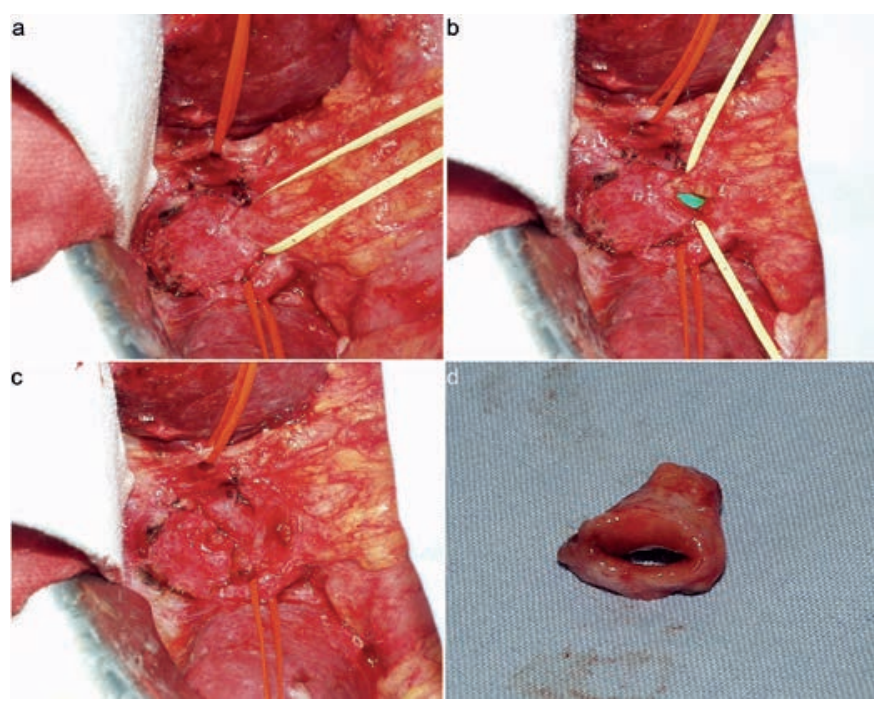

Fig. 6. Important steps in the surgical repair of a biliary duct laceration or insufficient anastomosis: preoperative control of the septic focus, use of vital tissue and removal of lacerated or ischemic tissue. a Biliary leak and secondary stenosis. b Identification of the leak (internal stenting by green plastic endostent). c Situs after resection of the ischemic area. d New anastomosis after resection of the ischemic biliary duct.

of eventual associated biloma. If a surgical re-exploration has to be performed, this should be postponed at least for 3-6 months if the clinical condition of the patient allows bridging by interventional techniques. The principles of delayed repair of the biliary system are depicted in figure 6 . They comprise preoperative control of the septic focus, use of vital tissue, and removal of lacerated or ischemic tissue.

Rare occasions of arrosion of the biliary anastomosis can result from pancreatic fistula after pancreatoduodenectomy. The theoretical countermeasure would be to drain fistula secretions away from the biliary anastomosis region. In our own practice, we use separate peritoneal drains for the biliary and pancreatic anastomosis at the primary operation. These conditions, when in place, do usually also require management of the pancreatic anastomosis, as reported there.

\section{Conclusion}

Bile leak after bilioenteric anastomosis is a rare condition that requires differentiated workup and therapy. Early leaks usually result from technical problems and are amenable to repair by revisional surgery and reoperation. Technical aspects and pitfalls have been discussed. The treatment of choice for late bile leaks is non-surgical.

\section{Disclosure Statement}

The authors have no conflict of interest to declare. 


\section{References}

1 Heidenhain C, Rosch R, Neumann UP: Hepatobiliary anastomosis techniques (Article in German). Chirurg 2011;82:7-10, 12-13.

2 Goessmann H, Lang SA, Fichtner-Feigl S, et al: Biliodigestive anastomosis: indications, complications and interdisciplinary management (Article in German). Chirurg 2012;83:1097-1108

3 Koch M, Garden OJ, Padbury R, et al: Bile leakage after hepatobiliary and pancreatic surgery: a definition and grading of severity by the International Study Group of Liver Surgery. Surgery 2011;149:680-688.

4 Linke R, Ulrich F, Bechstein WO, Schnitzbauer AA The White-test helps to reduce biliary leakage in liver resection: a systematic review and meta-analysis. Ann Hepatol 2015;14:161-167.
5 Klaiber U, Probst P, Knebel P, et al: Meta-analysis of complication rates for single-loop versus dual-loop (Roux-en-Y) with isolated pancreaticojejunostomy reconstruction after pancreaticoduodenectomy. $\mathrm{Br} \mathrm{J}$ Surg 2015;102:331-340.

6 Olthof PB, Coelen RJS, Wiggers JK, et al: External biliary drainage following major liver resection for perihilar cholangiocarcinoma: impact on development of liver failure and biliary leakage. HPB (Oxford) 2016;18: 348-353.

7 Neuhaus H, Hoffmann W, Classen M: The benefits and risks of percutaneous transhepatic cholangioscopy (Article in German). Dtsch Med Wochenschr 1993; 118:574-581.

8 Marin D, Bova V, Agnello F, et al: Gadoxetate disodium-enhanced magnetic resonance cholangiography for the noninvasive detection of an active bile duct leak after laparoscopic cholecystectomy. J Comput Assist Tomogr 2010;34:213-216.
9 Karavias D, Maroulis I, Karavias D: Intraoperative bile leakage detection during liver surgery using propofol. Ann R Coll Surg Engl 2014;96:628.

10 Mercado MA, Vilatobá M, Chan C, et al: Intrahepatic bilioenteric anastomosis after biliary complications of liver transplantation: operative rescue of surgical failures. World J Surg 2009;33:534-538.

11 Knorr C, Hohenberger W, Matzel KE, Kastl S: Inspection jejunostomy after complex reconstruction of the central bile duct. Indication, description, and personal experience (Article in German). Chirurg 2005;76:575580

12 Biglari M, Van den Bussche D, Vanlangenhove P: Reconstruction of a common bile duct injury by venous bypass. Acta Chir Belg 2013;113:308-310. 